\title{
Encryption Schemes Secure under Related-Key and Key-Dependent Message Attacks
}

\author{
Florian Böhl ${ }^{1, \star}$, Gareth T. Davies ${ }^{2, \star \star}$, and Dennis Hofheinz ${ }^{1, \star \star \star}$ \\ ${ }^{1}$ Karlsruhe Institute of Technology (KIT) \\ ${ }^{2}$ University of Bristol
}

\begin{abstract}
We construct secret-key encryption (SKE) schemes that are secure against related-key attacks and in the presence of key-dependent messages (RKA-KDM secure). We emphasize that RKA-KDM security is not merely the conjunction of individual security properties, but covers attacks in which ciphertexts of key-dependent messages under related keys are available. Besides being interesting in their own right, RKAKDM secure schemes allow to garble circuits with XORs very efficiently (Applebaum, TCC 2013). Until now, the only known RKA-KDM secure SKE scheme (due to Applebaum) is based on the LPN assumption. Our schemes are based on various other computational assumptions, namely DDH, LWE, QR, and DCR.

We abstract from Applebaum's construction and proof, and formalize three generic technical properties that imply RKA-KDM security: one property is IND-CPA security, and the other two are the existence of suitable oracles that produce ciphertexts under related keys, resp. of key-dependent messages. We then give simple SKE schemes that achieve these properties. Our constructions are variants of known KDM-secure public-key encryption schemes. To additionally achieve RKA security, we isolate suitable homomorphic properties of the underlying schemes in order to simulate ciphertexts under related keys in the security proof. RKA-KDM security for our schemes holds w.r.t. affine functions (over the respective mathematical domain).

From a conceptual point of view, our work provides a generic and extensible way to construct encryption schemes with multiple special security properties.
\end{abstract}

Keywords: related key attacks, key-dependent message security, garbled circuits.

\section{Introduction}

Motivation and Overview. The standard notion of security for secret-key encryption (SKE) is indistinguishability of ciphertexts (short: IND-CPA or IND-CCA, depending on whether passive or active attacks are considered).

\footnotetext{
* Supported by MWK grant "MoSeS".

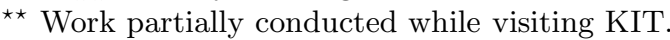

$\star \star \star$ Supported by DFG grant GZ HO 4534/2-1.

H. Krawczyk (Ed.): PKC 2014, LNCS 8383, pp. 483-500, 2014.

(C) International Association for Cryptologic Research 2014
} 
However, in certain applications, ciphertext indistinguishability is not sufficient. For instance, in harddisk encryption, encryptions of the secret key itself naturally occur (see 25]). Security in the presence of such key-dependent messages (KDM security [23]) is not implied by IND-CPA or IND-CCA security [23, 1]. There are numerous other specialized notions of encryption scheme security, such as security under related-key attacks (RKAs 7]), leakage-resilience [35, 29], security under bad randomness [10], security under selective openings [11], and others.

In this paper, we consider two such specialized notions of security for SKE schemes in a combined fashion. In particular, we will derive SKE schemes that are secure in the presence of key-dependent messages encrypted under related keys. This notion, dubbed RKA-KDM security and already considered by Applebaum [3] (as RK-KDM security), combines the notions of KDM and RKA security, but is more than just their conjunction. RKA-KDM secure SKE schemes are of course suitable for all applications in which RKA or KDM security is required. In fact, there are even applications that explicitly require the combined RKA-KDM notion: Applebaum [3] uses RKA-KDM secure SKE schemes in a garbled circuit construction in which XOR gates can be garbled for free (in the sense that XOR gates require no explicit encryption whatsoever). Besides, "aggregating" security properties as in RKA-KDM security may eventually lead to more "ideal" and universally useful security notions and encryption schemes.

RKA and KDM Security. To give more details, we first recall the definitions of IND-CPA, RKA, and KDM security. In a nutshell, an SKE scheme has indistinguishable ciphertexts (or, is IND-CPA secure 30]), if no efficient adversary $\mathcal{A}$ can tell apart whether it is interacting with an oracle Real, or with an oracle Fake. Here, upon input $M$, oracle Real returns an encryption $\mathrm{E}_{k}(M)$ of $M$, while Fake returns an encryption $\mathrm{E}_{k}\left(0^{|M|}\right)$ of a zero-string of the same length. (In other words, $\mathcal{A}$ is asked to tell authentic encryptions from encryptions of meaningless messages of the same length.)

For security under key-dependent messages (KDM security [23]), we require the same, except that messages are now functions in the secret key. That is, upon input a function $\psi$, Real returns $\mathrm{E}_{k}(\psi(k))$, and Fake returns $\mathrm{E}_{k}\left(0^{|\psi(k)|}\right)$. Depending on the class of allowed functions $\Psi$, there are many constructions of KDM-secure encryption schemes from various computational assumptions, e.g. [23, 31, 33, 25, 5, 28, 66, 26, 27, 34, 8, 12, 4, 32. However, most of these works follow the design principle of Boneh et al. [25] (henceforth BHHO). Namely, it should be publicly possible (or at least given some "harmless" extra information) to construct key-dependent encryptions from regular ones. Intuitively, if this is the case, then clearly the presence of key-dependent encryptions is no more harmful than the presence of "regular", key-independent encryptions.

For security under related-key attacks (RKA security [9]), we again require the same as for IND-CPA security, except that an adversary $\mathcal{A}$ now specifies a function $\varphi$ on secret keys alongside each message $M$ to be encrypted.

${ }^{1}$ In the following, for ease of exposition, we describe a modified but equivalent version of IND-CPA security. 
Real then returns an encryption $\mathrm{E}_{\varphi(k)}(M)$ of $M$ under the related key $\varphi(k)$, and Fake returns $\mathrm{E}_{\varphi(k)}\left(0^{|M|}\right)$. RKA security draws its motivation primarily from the wide range of attacks that are known in this setting, e.g. [16, 17, 18, 19, 21, 20, 22]. There are also a number of constructions of RKA secure schemes, e.g. [7, 13, 36, 3]. As with KDM security, the main idea is to generate encryptions under related keys from "regular" encryptions.

RKA-KDM Security. It is of course easy to combine RKA and KDM security into a combined notion, which we call RKA-KDM security here. Concretely, RKA-KDM security is defined like IND-CPA security above, only that an adversary supplies functions $\varphi$ and $\psi$ along with the message $M$ to be encrypted. Then, Real returns $\mathrm{E}_{\varphi(k)}(\psi(k))$, and Fake returns $\mathrm{E}_{\varphi(k)}\left(0^{|\psi(k)|}\right)$. This notion has already been defined by Applebaum [3] (dubbed RK-KDM security there), who used RKA-KDM secure schemes to garble circuits with XOR gates in a very elegant and efficient way. As a proof of concept, Applebaum also constructed an RKA-KDM secure encryption scheme, starting from the KDM-secure scheme of Applebaum et al. 5] based on the LPN assumption. (Along the way, he also shows that RKA-KDM security is strictly stronger than the conjunction of RKA and KDM security.) Currently, no further RKA-KDM secure schemes are known.

Our Contribution. In this work, we provide a generic framework to construct RKA-KDM secure encryption schemes, and we instantiate this framework under several computational assumptions. In particular, we provide RKA-KDM secure schemes from the decisional Diffie-Hellman (DDH), learning with errors (LWE), quadratic residuosity and decisional Diffie-Hellman (QR+DDH) 2 , and decisional composite residuosity (DCR) assumptions. Our constructions support affine KDM and RKA functions in the "natural domain" of the respective secret keys. Furthermore, with the exception of the DCR-based scheme, all of our schemes can be directly used in the application of Applebaum [3]. Additionally, they fit the construction of Bellare et al. [14], and thus can be extended from projection-KDM security to bounded-KDM security while maintaining the same level of RKA security.

Our Approach. Based on an informal remark of Applebaum [3, Remark 3.6 in full version], we first reduce RKA-KDM security to three technical properties of the scheme in question:

(a) IND-CPA security in the usual sense,

(b) the existence of an oracle (that itself has access to an $E_{k}(\cdot)$ oracle) that generates ciphertexts $\mathrm{E}_{\varphi(k)}(M)$ under related keys, and

(c) the existence of an oracle (with access to $\mathrm{E}_{k}(\cdot)$ ) that generates ciphertexts $\mathrm{E}_{k}(\psi(k))$ of key-dependent messages.

Intuitively, property (b) allows to reduce any RKA-KDM attack to a KDM attack, which in turn can be reduced (using (C) ) to an IND-CPA attack. We

${ }^{2}$ Similar to Hofheinz [32], we have to use the DDH assumption in the group of quadratic residues modulo $N$. 
note that it seems possible to add further oracles (e.g., for encryption queries with leakage) to achieve even stronger combined security notions from individual and isolated technical properties.

We then proceed to construct several RKA-KDM secure encryption schemes. Our constructions are slight variations of the known KDM-secure schemes from [25, 5, 6, 26, 34]. For these schemes, properties (国) and (ㄷ) already follow (with slight modifications) from the KDM security proofs of the underlying schemes. Showing property (b) then boils down to showing suitable homomorphic properties of the encryption, resp. decryption algorithm.

Example: Our DDH-Based Scheme. To give a taste of the proof, we outline our DDH-based scheme (which is based upon the DDH-based public-key encryption scheme from [25]). In this scheme, a ciphertext is of the form

$$
C=\left(g_{1}^{r_{1}}, \ldots, g_{\lambda}^{r_{\lambda}}, g^{M} \cdot g_{0}\right),
$$

where $\lambda$ is the security parameter, $g$ and the $g_{i}$ are uniformly random generators of the underlying cyclic group, the $r_{i}$ are uniformly random exponents, and $g_{0}=\prod_{i \in[\lambda]}\left(g_{i}^{r_{i}}\right)^{-k_{i}}$ for the secret key $k=\left(k_{1}, \ldots, k_{\lambda}\right) \in\{0,1\}^{\lambda}$. (In the original public-key encryption scheme from [25], all $r_{i}$ are identical.)

We show property (b) for functions of the form $\varphi_{\Delta}:\{0,1\}^{\lambda} \rightarrow\{0,1\}^{\lambda}$ with $\varphi_{\Delta}(k)=k \oplus \Delta$ for some $\Delta \in\{0,1\}^{\lambda}$. (This will be sufficient for the application in [3].) To show (b), we only need to show that any given ciphertext $C=\mathrm{E}_{k}(M)$ as above can be transformed into a ciphertext $C^{\prime}=\mathrm{E}_{\varphi_{\Delta}(k)}(M)$. For simplicity, assume that $\Delta=(1,0, \ldots, 0)$. In this case, it is easy to see that

$$
C^{\prime}=\left(1 / g_{1}^{r_{1}}, g_{2}^{r_{2}}, \ldots, g_{\lambda}^{r_{\lambda}},\left(g^{M} \cdot g_{0}\right) \cdot g_{1}^{r_{1}}\right)
$$

is a perfectly distributed encryption of $M$ under key $k^{\prime}=k \oplus \Delta$ (with randomness $r_{1}^{\prime}=-r_{1}$ and $r_{i}^{\prime}=r_{i}$ for $i>1$ ). This shows property (b) - the other properties follow as in [25] 3

Our other constructions proceed similarly, starting from the schemes of Applebaum et al. [5], Brakerski and Goldwasser [26], and Malkin et al. [34]. The latter is only contained in our full version [24].

\section{Preliminaries}

Notation. For $n \in \mathbb{N}$, let $[n]:=\{1, \ldots, n\}$. Throughout the paper, $\lambda \in \mathbb{N}$ denotes the security parameter. For a finite set $\mathcal{S}$, we denote by $s \leftarrow \mathcal{S}$ the process of sampling $s$ uniformly from $\mathcal{S}$. For a distribution $X$, we denote by $x \leftarrow X$ the process of sampling $x$ from $X$. For a probabilistic algorithm $A$, we denote with $y:=A(x ; r)$ the process of running $A$ on input $x$ and with

\footnotetext{
${ }^{3}$ We note that our technical change to the scheme from [25] - namely, using different $r_{i}$ - can be proven to be not crucial to its security (see Theorem 7). Instead, choosing different $r_{i}$ simplifies expressing the scheme in our framework, and in particular separating the KDM, RKA, and IND-CPA properties.
} 
randomness $r$, and assigning $y$ the result. We let $\mathcal{R}_{A}$ denote the randomness space of $A$; we require $\mathcal{R}_{A}$ to be of the form $\mathcal{R}_{A}=\{0,1\}^{\ell}$. We write $y \leftarrow A(x)$ for $y \leftarrow A(x ; r)$ with uniformly chosen $r \in \mathcal{R}_{A}$. If $A$ 's running time is polynomial in $\lambda$, then $A$ is called probabilistic polynomial-time (PPT). For a real number $x$, let the floor function $\lfloor x\rfloor$ denote the largest integer not greater than $x$. For a vector $\mathbf{v}, \mathbf{v}_{i}$ denotes the $i$ th element of $\mathbf{v}$.

Two sequences of random variables $X=\left(X_{\lambda}\right)_{\lambda \in \mathbb{N}}$ and $Y=\left(Y_{\lambda}\right)_{\lambda \in \mathbb{N}}$ are computationally indistinguishable (denoted $X \stackrel{c}{\approx} Y$ ) iff for any PPT algorithm $D$, the probability $\operatorname{Pr}\left[D\left(1^{\lambda}, X_{\lambda}\right)=1\right]-\operatorname{Pr}\left[D\left(1^{\lambda}, Y_{\lambda}\right)\right]$ is negligible in $\lambda . X=$ $\left(X_{\lambda}\right)_{\lambda \in \mathbb{N}}$ and $Y=\left(Y_{\lambda}\right)_{\lambda \in \mathbb{N}}$ are statistically indistinguishable (denoted $X \stackrel{s}{\approx} Y$ ) iff the same holds for any algorithm $D$ with unbounded runtime.

SKE Schemes. A secret-key encryption (SKE) scheme consists of four PPT algorithms (Pg, Kg, E, D). Parameter generation $\mathrm{Pg}\left(1^{\lambda}\right)$ outputs public parameters $\pi$ for the scheme. Key generation $\mathrm{Kg}(\pi)$ outputs a (secret) key $k$. Encryption $\mathrm{E}_{k}(M)$ takes a key $k$ and a message $M$, and outputs a ciphertext $C$. Decryption $\operatorname{Dec}_{k}(C)$ takes a key $k$ and a ciphertext $C$, and outputs a message $M$ or $\perp$ if decryption fails. For correctness, we stipulate $\mathrm{D}_{k}(C)=M$ for all $M$, all $k \leftarrow \operatorname{Kg}\left(\operatorname{Pg}\left(1^{\lambda}\right)\right)$, and all $C \leftarrow \mathrm{E}_{k}(M)$.

Definition 1 (RKA-KDM $[\Phi, \Psi]$ Security.). Let $\Sigma=(\mathrm{Pg}, \mathrm{Kg}, \mathrm{E}, \mathrm{D})$ be a symmetric encryption scheme, $\pi \leftarrow \operatorname{Pg}\left(1^{\lambda}\right)$ be public parameters and $b \leftarrow\{0,1\}$ be a bit chosen by the challenger. A key $k \leftarrow \mathrm{Kg}(\pi)$ is randomly chosen. Adversary $\mathcal{A}$ makes encryption queries by submitting $(\varphi \in \Phi, \psi \in \Psi)$ and receives a response from one of the following oracles, depending on the bit $b$.

- If $b=1$, oracle Real $_{k}$ takes as input $(\varphi, \psi)$ and returns $C \leftarrow \mathrm{E}_{\varphi(k)}(\psi(k))$.

- If $b=0$, oracle Fake $_{k}$ takes as input $(\varphi, \psi)$ and returns $C \leftarrow \mathrm{E}_{\varphi(k)}\left(0^{|\psi(k)|}\right)$.

Scheme $\Sigma$ is RKA-KDM secure w.r.t. $\Phi$ and $\Psi$ if for all PPT adversaries $\mathcal{A}$

$$
\left|\operatorname{Pr}\left[\mathcal{A}^{\operatorname{Real}(\varphi, \psi)}(\pi)=1\right]-\operatorname{Pr}\left[\mathcal{A}^{\operatorname{Fake}(\varphi, \psi)}(\pi)=1\right]\right|
$$

is a negligible function in $\lambda$.

Throughout this paper each class of KDM functions $\Psi$ implicitly contains constant functions $\psi_{M}(k):=M$ for all messages $M \in \mathcal{M}$ where $\mathcal{M}$ is the message space of the encryption scheme at hand.

Further Security Definitions. The standard definition of $R K A$ security follows from restricting the KDM function class $\Psi$ to constant functions, and the definition of KDM security follows from restricting the RKA function class $\Phi$ to the identity function. IND-CPA security follows from applying both of these restrictions at once. 


\subsection{A Generic Approach}

In this section we prove that an SKE scheme $\Sigma$ is $\operatorname{RKA-KDM}[\Phi, \Psi]$ secure if $-\Sigma$ is IND-CPA secure,

- there is a so called RKA $[\Phi]$ oracle (defined below) for $\Sigma$ that takes as input $\mathrm{E}_{k}(M)$ and RKA function $\varphi \in \Phi$, and returns something that is indistinguishable from $\mathrm{E}_{\varphi(k)}(M)$ without knowledge of the key $k$,

- there is a so called $\operatorname{KDM}[\Psi]$ oracle (defined below) for $\Sigma$ that takes as input $\mathrm{E}_{k}(M)$ and $\mathrm{KDM}$ function $\psi \in \Psi$, and returns something that is indistinguishable from $\mathrm{E}_{k}(\psi(k))$ without knowledge of the key $k$ ( $M$ is the constant part of $\psi$ here).

Definition 2 (RKA $[\Phi]$ oracle). Let $\Sigma=(\mathrm{Pg}, \mathrm{Kg}, \mathrm{E}, \mathrm{D})$ be a secret key encryption scheme with message space $\mathcal{M}$. We say that a function $\mathcal{F}_{\mathrm{RKA}[\Phi]}(\varphi, C)$ is an RKA $[\Phi]$ oracle for $\Sigma$ iff for all PPT adversaries $\mathcal{A}$ that make queries $(\varphi, M)$ for $\varphi \in \Phi$ and $M \in \mathcal{M}$

$$
\begin{aligned}
& \mid \operatorname{Pr}\left[\mathcal{A}^{\mathcal{F}_{\mathrm{RKA}[\Phi]}\left(\varphi, \mathrm{E}_{k}(\cdot)\right)}(\pi, k)=1: \pi \leftarrow \operatorname{Pg}\left(1^{\lambda}\right), k \leftarrow \operatorname{Kg}(\pi)\right] \\
& -\operatorname{Pr}\left[\mathcal{A}^{\mathrm{E}_{\varphi(k)}(\cdot)}(\pi, k)=1: \pi \leftarrow \operatorname{Pg}\left(1^{\lambda}\right), k \leftarrow \operatorname{Kg}(\pi)\right] \mid
\end{aligned}
$$

is a negligible function in $\lambda$. Here, $\mathcal{A}^{\mathcal{F}_{\mathrm{RKA}[\Phi]}\left(\varphi, \mathrm{E}_{k}(\cdot)\right)}$ denote the interaction of $\mathcal{A}$ with an oracle that, upon input $M$, outputs $\mathcal{F}_{\mathrm{RKA}[\Phi]}\left(\varphi, \mathrm{E}_{k}(M)\right)$.

Definition 3 (KDM $[\Psi]$ oracle). Let $\Sigma=(\mathrm{Pg}, \mathrm{Kg}, \mathrm{E}, \mathrm{D})$ be a secret key encryption scheme with message space $\mathcal{M}$. We say that a function $\mathcal{F}_{\mathrm{KDM}[\Psi]}(\psi, C)$ is a $\operatorname{KDM}[\Psi]$ oracle for $\Sigma$ iff for all PPT adversaries $\mathcal{A}$ that make queries $\psi$ for $\psi \in \Psi$ (where $M$ denotes the constant part of $\psi$, i.e., $\psi(0)$ )

$$
\begin{aligned}
& \mid \operatorname{Pr}\left[\mathcal{A}^{\mathcal{F}_{\mathrm{KDM}[\Psi]}\left(\psi, \mathrm{E}_{k}(M)\right)}(\pi, k)=1: \pi \leftarrow \operatorname{Pg}\left(1^{\lambda}\right), k \leftarrow \mathrm{Kg}(\pi)\right] \\
& -\operatorname{Pr}\left[\mathcal{A}^{\mathrm{E}_{k}(\psi(k))}(\pi, k)=1: \pi \leftarrow \operatorname{Pg}\left(1^{\lambda}\right), k \leftarrow \operatorname{Kg}(\pi)\right] \mid
\end{aligned}
$$

is a negligible function in $\lambda$.

Note that for constant functions $\psi \in \Psi$ a sufficient behaviour of $\mathcal{F}_{\mathrm{KDM}[\Psi]}$ is to output the ciphertext it received without changes. All $\mathrm{KDM}[\Psi]$ oracles presented in this paper implicitly adopt this behaviour.

Theorem 4. Let $\Sigma$ be an SKE scheme that is IND-CPA secure, $\mathcal{F}_{\mathrm{RKA}[\Phi]}$ be an $\mathrm{RKA}[\Phi]$ oracle for $\Sigma$ and $\mathcal{F}_{\mathrm{KDM}[\Psi]}$ be a $\mathrm{KDM}[\Psi]$ oracle for $\Sigma$. Then $\Sigma$ is $\operatorname{RKA-KDM}[\Phi, \Psi]$ secure.

Proof. We prove the theorem by a sequence of games.

Game 0. In Game $0 \mathcal{A}$ plays the original RKA-KDM $[\Phi, \Psi]$ experiment (see Theorem 1). 
Game 1. In Game1, instead of computing $\mathrm{E}_{\varphi(k)}(\psi(k))$ the experiment computes $C_{\mathrm{KDM}} \leftarrow \mathrm{E}_{k}(\psi(k))$ and outputs $\mathcal{F}_{\mathrm{RKA}[\Phi]}\left(\varphi, C_{\mathrm{KDM}}\right)$ to the adversary. This game is indistinguishable from Game 0 due to the indistinguishability of $\mathcal{F}_{\mathrm{RKA}[\Phi]}$ (see Theorem 2).

Game 2. In Game 2] instead of computing $\mathrm{E}_{k}(\psi(k))$, the experiment computes $C_{\mathrm{CPA}} \leftarrow \mathrm{E}_{k}(M)$ where $M$ is the constant part of $\psi$ and sets $C_{\mathrm{KDM}}:=$ $\mathcal{F}_{\mathrm{KDM}[\Psi]}\left(\psi, C_{\mathrm{CPA}}\right)$. Given a distinguisher $\mathcal{D}$ between this game and Game 1, we can construct an adversary $\mathcal{S}$, henceforth called simulator, on the indistinguishability of $\mathcal{F}_{\mathrm{KDM}[\Psi]}$. First, the simulator forwards the public parameters $\pi$ to $\mathcal{D}$ and picks a bit $b \leftarrow\{0,1\}$. For $b=1$ and each query $(\varphi, \psi)$ from $\mathcal{D}$, the simulator queries its oracle for $\psi$ and either gets a response $\mathcal{F}_{\mathrm{KDM}[\Psi]}\left(\psi_{M}, C_{\mathrm{CPA}}\right)$ or $\mathrm{E}_{k}\left(\psi_{M}(k)\right)$ (see Theorem 3). It then applies $\mathcal{F}_{\mathrm{RKA}[\Phi]}$ with $\varphi$ to the response and sends the result to $\mathcal{D}$. The responses to the queries of the simulator are that of Game 2 if itself gets responses of type $\mathcal{F}_{\mathrm{KDM}[\Psi]}\left(\psi_{M}, C_{\mathrm{CPA}}\right)$ and that of Game 1 for responses of type $\mathrm{E}_{k}\left(\psi_{M}(k)\right)$. Analogously for $b=0$, where the simulator queries $0^{|\psi(k)|}$ instead of $\psi$. The advantage of $\mathcal{S}$ is that of $\mathcal{D}$ and must be negligible due to the indistinguishability $\mathcal{F}_{\mathrm{KDM}[\Psi]}$.

Game 3. In Game 3 we replace $C_{\mathrm{CPA}} \leftarrow \mathrm{E}_{k}(M)$ by $C_{\mathrm{CPA}} \leftarrow \mathrm{E}_{k}\left(0^{|M|}\right)$. Analogously to the indistinguishability of Game 1 and Game 2, we can easily transform a distinguisher between this game and the previous game into an IND-CPA adversary for $\Sigma$.

We observe that the advantage of any PPT adversary in Game 3 is 0 since the behaviour of the oracle given to the adversary is is independent of the bit $b$ picked by the experiment. This concludes our proof since Game 3 and Game 0 are indistinguishable.

\section{RKA-KDM-Secure Encryption Schemes}

\subsection{Boneh et al. [25]}

The PKE scheme of Boneh et al. 25] was the first construction provably KDM secure under standard assumptions. In this section we detail a SKE analogue of the 'basic' version of their scheme. We construct an RKA $[\Phi]$ oracle and a $\operatorname{KDM}[\Psi]$ oracle for the scheme. The class of RKA functions $\Phi$ allows for XOR operations on the key while the class of KDM functions $\Psi$ brings circular KDM security, i.e., encryptions of the secret key are possible (as in the original paper). The security of the scheme is based on the DDH assumption.

DDH Assumption. The decisional Diffie-Hellman (DDH) assumption over a group $\mathbb{G}$ (that may depend on the security parameter $\lambda$ ) stipulates that

$$
\left(g, g^{x}, g^{y}, g^{x y}\right) \stackrel{c}{\approx}\left(g, g^{x}, g^{y}, g^{z}\right)
$$

where $g \leftarrow \mathbb{G}$ and $x, y, z \leftarrow[|\mathbb{G}|]$ are uniformly distributed. 
For the sake of readability we introduce the scheme $\Sigma_{\mathrm{BHHO}}^{\prime}$ with message space $\{0,1\}$. Canonical concatenation at the end will yield the scheme $\Sigma_{\text {BHHO }}$ with message space $\{0,1\}^{\lambda}$.

The SKE Scheme $\boldsymbol{\Sigma}_{\text {BнHO }}^{\prime}$ Let $\mathbb{G}$ be a group of prime order $p$ and $g$ be a generator of $\mathbb{G}$. The scheme $\Sigma_{\mathrm{BHHO}}^{\prime}$ for $M \in\{0,1\}$ is defined as follows:

- $\operatorname{Pg}\left(1^{\lambda}\right)$ picks generators $g_{1}, \ldots, g_{\lambda} \leftarrow \mathbb{G} \backslash\{1\}$ and returns $\pi:=\left(\mathbb{G}, g, g_{1}, \ldots, g_{\lambda}\right)$.

- $\operatorname{Kg}(\pi)$ returns a random bitstring $k \leftarrow\{0,1\}^{\lambda}$.

$-\mathrm{E}_{k}(M)$ picks $r_{1}, \ldots, r_{\lambda} \leftarrow \mathbb{Z}_{p}$. Sets $g_{0}:=\prod_{i \in[\lambda]}\left(g_{i}^{r_{i}}\right)^{-k_{i}}$ and returns

$$
C:=\left(g_{1}^{r_{1}}, \ldots, g_{\lambda}^{r_{\lambda}}, g^{M} \cdot g_{0}\right) \in \mathbb{G}^{\lambda+1} .
$$

- $\mathrm{D}_{k}(C)$ parses $C$ as $\left(x_{1}, \ldots, x_{\lambda}, y\right)$. Computes $\tilde{M}:=y \cdot \prod_{i \in[\lambda]} x_{i}^{k_{i}}$. Returns 0 if $\tilde{M}=1$, returns 1 if $\tilde{M}=g$, otherwise returns $\perp$.

The RKA $[\boldsymbol{\Phi}]$ Oracle. For the concrete class of RKA functions

$$
\Phi:=\left\{\varphi_{\Delta}:\{0,1\}^{\lambda} \rightarrow\{0,1\}^{\lambda}, k \mapsto k \oplus \Delta: \Delta \in\{0,1\}^{\lambda}\right\}
$$

we find an RKA $[\Phi]$ oracle $\mathcal{F}_{\mathrm{RKA}[\Phi]}$ for $\Sigma_{\mathrm{BHHO}}^{\prime}$ as follows: Given a ciphertext $C=$ $\left(x_{1}, \ldots, x_{\lambda}, y\right)$ and a function $\varphi_{\Delta}$ it outputs

$$
C^{\prime}:=\left(x_{1}^{\prime}, \ldots, x_{\lambda}^{\prime}, y^{\prime}\right):=\left(x_{1}^{(-1)^{\Delta_{1}}}, \ldots, x_{\lambda}^{(-1)^{\Delta_{\lambda}}}, y \cdot \prod_{i \in[\lambda]} x_{i}^{\Delta_{i}}\right)
$$

To understand this better we assume that $C$ is an honestly generated ciphertext (as it will be in the indistinguishability experiment for $\mathcal{F}_{\mathrm{RKA}[\Phi]}$ ). Then we have $y=g^{M} \cdot \prod_{i \in[\lambda]} x_{i}^{-k_{i}}$. We observe

$y^{\prime}=g^{M} \cdot \prod_{i \in[\lambda]} x_{i}^{-k_{i}} \cdot \prod_{i \in[\lambda]} x_{i}^{\Delta_{i}}=g^{M} \cdot \prod_{i \in[\lambda]} x_{i}^{(-1)^{\Delta_{i}}\left(-k_{i}+\Delta_{i}\right) \stackrel{(*)}{=}} g^{M} \cdot \prod_{i \in[\lambda]} x_{i}^{\prime-\left(k_{i} \oplus \Delta_{i}\right)}$

and $(*)$ since

$$
(-1)^{\Delta_{i}}\left(-k_{i}+\Delta_{i}\right)=\left\{\begin{aligned}
-k_{i} & \text { if } \Delta_{i}=0 \\
-\left(1-k_{i}\right) & \text { if } \Delta_{i}=1
\end{aligned}\right\}=-\left(k_{i} \oplus \Delta_{i}\right)
$$

Therefore $C^{\prime}$ decrypts to $M$ under key $k \oplus \Delta$.

Lemma 5. $\mathcal{F}_{\mathrm{RKA}[\Phi]}$ is an $\mathrm{RKA}[\Phi]$ oracle in the sense of Theorem 2.

Proof. It is easy to see that the distributions of $\mathcal{F}_{\mathrm{RKA}[\Phi]}\left(\varphi_{\Delta}, \mathrm{E}_{k}(M)\right)$ and $\mathrm{E}_{k \oplus \Delta}(M)$ are perfectly indistinguishable (even for someone knowing $k$ and $\Delta$ ): The $x_{i}^{\prime}$ just look like $r_{i}^{\prime}=(-1)^{\Delta_{i}} r_{i}$ was used as randomness for the $i$ th component (which yields the same distribution) and we have $y^{\prime}=g^{M} \cdot \prod_{i \in[\lambda]}\left(x_{i}^{\prime}\right)^{-\left(k_{i} \oplus \Delta_{i}\right)}$. 
The KDM $\left[\Psi^{\prime}\right]$ Oracle. For the class of KDM functions

$$
\Psi^{\prime}:=\left\{\psi_{i, b}:\{0,1\}^{\lambda} \rightarrow\{0,1\}, k \mapsto k_{i} \oplus b: i \in[\lambda], b \in\{0,1\}\right\}
$$

we find the following $\mathrm{KDM}\left[\Psi^{\prime}\right]$ oracle $\mathcal{F}_{\mathrm{KDM}\left[\Psi^{\prime}\right]}$ for $\Sigma_{\mathrm{BHHO}}^{\prime}$ : Given a function $\psi_{i, b}$ and an honestly generated ciphertext of $b$ (the constant part of $\psi_{i, b}$ is $b$ ) denoted $C=\left(x_{1}, \ldots, x_{\lambda}, y\right)$ it outputs

$$
C^{\prime}:=\left(x_{1}^{\prime}, \ldots, x_{\lambda}^{\prime}, y^{\prime}\right):=\left(x_{1}, \ldots, x_{i-1}, x_{i} \cdot g^{(-1)^{b}}, x_{i+1}, \ldots, x_{\lambda}, y\right)
$$

We check that this ciphertext decrypts to $k_{i} \oplus b$ :

$y \cdot \prod_{j \in[\lambda]} x_{j}^{\prime k_{j}} \stackrel{(*)}{=} y \cdot\left(\prod_{j \in[\lambda]} x_{j}^{k_{j}}\right) \cdot g^{(-1)^{b} \cdot k_{i}}=g^{b} \cdot\left(\prod_{j \in[\lambda]} x_{j}^{-k_{j}} \cdot x_{j}{ }^{k_{j}}\right) \cdot g^{(-1)^{b} \cdot k_{i}}=g^{k_{i} \oplus b}$

(*) since $x_{i}^{\prime}=x_{i} \cdot g^{(-1)^{b}}$ and $x_{j}^{\prime}=x_{j}$ for $j \in[\lambda] \backslash\{i\}$.

Lemma 6. $\mathcal{F}_{\mathrm{KDM}\left[\Psi^{\prime}\right]}$ is a $\mathrm{KDM}\left[\Psi^{\prime}\right]$ oracle in the sense of Theorem 3.

Proof. We show that the distributions of $\mathcal{F}_{\mathrm{KDM}\left[\Psi^{\prime}\right]}\left(\psi_{i, b}, \mathrm{E}_{k}(b)\right)$ and $\mathrm{E}_{k}\left(\psi_{i, b}(k)\right)$ are perfectly indistinguishable. First, we observe that $x_{i}=g_{i}^{r_{i}}$ and $g=g_{i}^{\alpha}$ for $\alpha:=\log _{g_{i}}(g)$, i.e., $x_{i}^{\prime}=g^{r_{i}+(-1)^{b} \cdot \alpha}$. Furthermore we have $y=g^{b} \cdot \prod_{j \in[\lambda]} x_{j}{ }^{-k_{j}}=$ $g^{b} \cdot \prod_{j \in[\lambda]} x_{j}^{-k_{j}} g^{-(-1)^{b} \cdot k_{i}} g^{(-1)^{b} \cdot k_{i}}=g^{b+(-1)^{b}} \cdot \prod_{j \in[\lambda]} x_{j}^{\prime-k_{j}}$. Hence the output of the oracle looks like a normal encryption of $k_{i} \oplus b$ where $r_{i}+(-1)^{b} \cdot \alpha$ was used as randomness in the $i$ th component.

Lemma 7. The SKE scheme $\Sigma_{\mathrm{BHHO}}^{\prime}$ is IND-CPA secure if DDH is hard over the underlying group $\mathbb{G}$.

Proof. Intuitively, we first use the hardness of DDH over $\mathbb{G}$ to collapse the randomness used by the encryption oracle to one random exponent per ciphertext, so instead of $r_{1}, \ldots, r_{\lambda}$ all generators are taken to the same random exponent $r$. This modified scheme is the 'basic' version of [25] with a smaller message space. We can then simply reduce security to the IND-CPA security of Boneh et al's scheme.

More concretely, we prove the lemma with the following sequence of games.

Game 0. In Game $0 \mathcal{A}$ plays the original IND-CPA experiment.

Game 1 to Game $\lambda-1$ form a hybrid argument to collapse the randomness used by the encryption oracle. In hybrid $i(i \in[\lambda-1])$ we pick the same randomness for the first $i+1$ components of the ciphertext. I.e., the format of a ciphertext output by the encryption oracle in game $i$ is

$$
\left(g_{1}^{r}, \ldots, g_{i+1}^{r}, g_{i+2}^{r_{i+2}}, \ldots, g_{\lambda}^{r_{\lambda}}, g^{M} \cdot\left(\prod_{i \in[i+1]} g_{i}^{-r k_{i}}\right)\left(\prod_{i \in[\lambda] \backslash[i+1]} g_{i}^{-r_{i} k_{i}}\right)\right)
$$


Analysis. Each of the game hops above is indistinguishable due to the hardness of DDH over $\mathbb{G}$. The simulation for a hop from Game $i-1$ to Game $i(i \in[\lambda-1])$ works as follows: The simulator $\mathcal{S}$ gets a DDH challenge $\left(g, X:=g^{x}, Y:=\right.$ $\left.g^{y}, Z:=g^{x y / z}\right)$. For $j \in[\lambda] \backslash\{i+1\}$ it picks $\alpha_{j} \leftarrow \mathbb{Z}_{p}$, sets $g_{j}:=g^{\alpha_{j}}$ and $g_{i+1}:=$ $X$. Subsequently it picks a key $k \leftarrow\{0,1\}^{\lambda}$ and sends the public parameters $\pi:=\left(\mathbb{G}, g, g_{1}, \ldots, g_{\lambda}\right)$ to $\mathcal{A}$. If $\mathcal{A}$ requests an encryption of message $M, \mathcal{S}$ picks randomness $r, r_{i+2}, \ldots, r_{\lambda}, a, b \leftarrow \mathbb{Z}_{p}$ and sets $\hat{Y}:=g^{a} \cdot Y^{b}$ and $\hat{Z}:=X^{a} \cdot Z^{b}$ to re-randomize the DDH challenge. Finally, $\mathcal{S}$ sends

$$
\left(\hat{Y}^{r \alpha_{1}}, \ldots, \hat{Y}^{r \alpha_{i}}, \hat{Z}^{r}, g_{i+2}^{r_{i+2}}, \ldots, g^{M} \cdot g_{0}\right)
$$

to the adversary where $g_{0}$ is computed as usual $(\mathcal{S}$ knows $k)$. If $Z=g^{z}$, the output of $\mathcal{S}$ looks like that of game $i-1$, otherwise (for $Z=g^{x y}$ ) it looks like that of game $i$. Any PPT distinguisher between those games with non-negligible advantage can thus be used to break DDH.

Finally, only one fresh random exponent is used for each ciphertext in game $\lambda-1$. The output now looks like that of the BHHO (public key) cryptosystem with message space $\left\{g^{0}, g^{1}\right\}$.

In Game $\lambda$, we replace the message with 0 . The indistinguishability of game $\lambda-1$ and game $\lambda$ can be reduced to the IND-CPA security of Boneh et al's original scheme in a straightforward way (using the generators from the public key as public parameters). Hence IND-CPA security of $\Sigma_{\mathrm{BHHO}}^{\prime}$ follows.

The Full Scheme $\boldsymbol{\Sigma}_{\text {Bнно }}$. Finally, we assemble the SKE scheme $\Sigma_{\text {Bнно from }}$ $\lambda$ instances of $\Sigma_{\mathrm{BHHO}}^{\prime}$ that use the same public parameters $\pi$ and the same key $k$. A ciphertext under $\Sigma_{\mathrm{BHHO}}$ is a matrix from $\mathbb{G}^{\lambda \times(\lambda+1)}$ where each row is an instance of $\Sigma_{\mathrm{BHHO}}^{\prime}$ (using $\pi$ and key $k$ ). To encrypt a message $M \in\{0,1\}^{\lambda}$ under key $k$ we encrypt $M_{i}$ in row $i$ (while picking fresh randomness $r_{i}, i \in[\lambda]$ for each row). Decryption also works row-wise.

For the $\operatorname{RKA}[\Phi]$ oracle we apply $\mathcal{F}_{\mathrm{RKA}[\Phi]}$ to each row. The class of KDM functions $\Psi^{\prime}$ changes to

$$
\Psi:=\left\{\psi_{\mathbf{i}, \Delta}:\{0,1\}^{\lambda} \rightarrow\{0,1\}^{\lambda}, k \mapsto\left(k_{\mathbf{i}_{1}} \oplus \Delta_{1}, \ldots, k_{\mathbf{i}_{\lambda}} \oplus \Delta_{\lambda}\right): \mathbf{i} \in[\lambda]^{\lambda}, \Delta \in\{0,1\}^{\lambda}\right\}
$$

I.e., each bit of the message can be an arbitrarily picked key bit. For the KDM $[\Psi]$ oracle provided with function $\psi_{\mathbf{i}}$, we apply $\mathcal{F}_{\mathrm{KDM}\left[\Psi^{\prime}\right]}$ with function $\psi_{\mathbf{i}_{j}} \in \Psi^{\prime}$ to the $j$ th row of the ciphertext where $\Psi^{\prime}$ is the class of KDM functions for $\Sigma_{\text {BHнO }}^{\prime}$. Since the oracles work row-wise it is easy to check that the indistinguishability results from Theorem 5 and Theorem 5 carry over to $\Sigma_{\mathrm{BHHO}}$. Analogously for the IND-CPA security of $\Sigma_{\mathrm{BHHO}}$. Finally, by Theorem 4 , we get

Theorem 8. The SKE scheme $\Sigma_{\mathrm{BHHO}}$ is $\operatorname{RKA-KDM}[\Phi, \Psi]$ secure (for $\Phi$ and $\Psi$ as defined above in this section) if DDH is hard over the underlying group $\mathbb{G}$.

\subsection{Applebaum et al. [5]}

In this section, we present a secret-key version of the PKE scheme of Applebaum et al. [5] and prove it RKA-KDM secure. For compatibility with Applebaum's 
application, however, we slightly change the space of secret keys from $\mathbb{Z}_{p}^{m}$ to $\{0,1\}^{m}$. Our RKA and KDM oracles allow encryptions under keys $k \oplus \Delta$ (for arbitrary $\Delta \in\{0,1\}^{m}$ ) of arbitrary components of the secret key. Security is based on the LWE assumption.

For ease of exposition, we do not detail the choices of the following parameters - these can occur as in [5] (with adaptations as in [2] due to the different choice of secret key). Let $q$ be a polynomial in the security parameter $\lambda$, and let $m>n$ be integers (that may also depend on $\lambda$ ). By $\chi$, we denote a (discretized Gaussian) error distribution with suitable parameters over $\mathbb{Z}_{q}$.

LWE Assumption. Let $\mathbf{s} \in \mathbb{Z}_{q}^{n}$ be uniformly chosen. Let $\operatorname{LWE}_{\mathbf{s}}$ be the oracle that (on trivial input) returns $(\mathbf{a},\langle\mathbf{a} ; \mathbf{s}\rangle+x) \in \mathbb{Z}_{q}^{n} \times \mathbb{Z}_{q}$ for freshly chosen $\mathbf{a} \leftarrow \mathbb{Z}_{q}^{n}$ and $x \leftarrow \chi$. Let RND be the oracle that returns a freshly and independently chosen $(\mathbf{a}, \mathbf{b}) \leftarrow \mathbb{Z}_{q}^{n} \times \mathbb{Z}_{q}$. The LWE assumption states that oracle access to $\mathrm{LWE}_{\mathbf{s}}$ is computationally indistinguishable from oracle access to RND.

Applebaum et al. [5] show that the LWE assumption over $\mathbb{Z}_{q}=\mathbb{Z}_{p^{2}}$ and with $\mathbf{s} \leftarrow \mathbb{Z}_{p}^{n}$ is equivalent to the LWE assumption as above (for $q=p$ ). Furthermore, Akavia et al. 22] show that the LWE assumption with $\mathbf{s} \leftarrow\{0,1\}^{n}$ is implied by the LWE assumption as above (for different parameters of $n, m$ ). In the following, we will consider $q=p^{2}$ and $\mathbf{s} \in\{0,1\}^{n}$. Furthermore, for $x \in \mathbb{R}$, we write $\lceil x\rfloor_{p}:=\lceil x+1 / 2\rceil \bmod p$ for the nearest integer to $x$ modulo $p$.

The SKE Scheme $\Sigma_{\text {ACPS }}^{\prime}$. The scheme $\Sigma_{\text {ACPS }}^{\prime}$ (with $M \in \mathbb{Z}_{p}$ ) is defined as follows:

$-\operatorname{Pg}\left(1^{\lambda}\right)$ returns the empty bitstring.

$-\operatorname{Kg}(\pi)$ returns a random bitstring $k:=\mathbf{s} \leftarrow\{0,1\}^{m}$.

$-\mathrm{E}_{k}(M)$ picks $\mathbf{A} \leftarrow \mathbb{Z}_{q}^{n \times m}$ and $\mathbf{r}, \mathbf{x} \leftarrow \chi^{m}$, and returns

$$
C:=\left(\mathbf{A} \cdot \mathbf{r},-\left(\mathbf{s}^{T} \cdot \mathbf{A}+\mathbf{x}^{T}\right) \cdot \mathbf{r}+p \cdot M\right)=\left(\mathbf{A} \cdot \mathbf{r},-\mathbf{s}^{T} \cdot \mathbf{A} \cdot \mathbf{r}-\langle\mathbf{x} ; \mathbf{r}\rangle+p \cdot M\right) \in \mathbb{Z}_{q}^{m} \times \mathbb{Z}_{q}
$$

- $\mathrm{D}_{k}(C)$ parses $C=:(\mathbf{y}, z)$ and computes and returns $M:=\lceil(\langle\mathbf{s} ; \mathbf{y}\rangle+z) / p\rfloor_{p}$.

Compared to the PKE scheme of [5], we choose s slightly differently, and also choose different $\mathbf{A}, \mathbf{x}$ upon each encryption. We note that correctness holds only with overwhelming probability over the choice of $\mathbf{r}$ and $\mathbf{x}$. In particular, $|\langle\mathbf{x} ; \mathbf{r}\rangle|<$ $p / 2$ with overwhelming probability.

The RKA $[\boldsymbol{\Phi}]$ Oracle. For the concrete class of RKA functions

$$
\Phi:=\left\{\varphi_{\Delta}:\{0,1\}^{m} \rightarrow\{0,1\}^{m}, k \mapsto k \oplus \Delta: \Delta \in\{0,1\}^{m}\right\},
$$

we find an $\operatorname{RKA}[\Phi]$ oracle $\mathcal{F}_{\mathrm{RKA}[\Phi]}$ for $\Sigma_{\text {ACPS }}^{\prime}$ as follows: Given a ciphertext $C=$ $(\mathbf{y}, z)$ and a function $\varphi_{\Delta}$, it outputs

$$
C^{\prime}:=\left(\mathbf{y}^{\prime}, z^{\prime}\right) \quad \text { with } \quad \mathbf{y}_{i}^{\prime}=(-1)^{\Delta_{i}} \mathbf{y}_{i} \quad \text { and } \quad z^{\prime}=z+\sum_{i \in[m]} \Delta_{i} \mathbf{y}_{i}
$$

As with the BHHO scheme, a quick calculation shows that $C^{\prime}$ is a perfectly distributed ciphertext of $M$ under $k \oplus \Delta$. Thus:

Lemma 9. $\mathcal{F}_{\mathrm{RKA}[\Phi]}$ is an $\mathrm{RKA}[\Phi]$ oracle in the sense of Theorem 2. 
The $\operatorname{KDM}\left[\boldsymbol{\Psi}^{\prime}\right]$ Oracle. For the class of KDM functions

$$
\Psi^{\prime}:=\left\{\psi_{i, b}:\{0,1\}^{\lambda} \rightarrow\{0,1\}, k \mapsto k_{i} \oplus b: i \in[\lambda], b \in\{0,1\}\right\}
$$

and following [5], we find the following $\operatorname{KDM}\left[\Psi^{\prime}\right]$ oracle $\mathcal{F}_{\mathrm{KDM}\left[\Psi^{\prime}\right]}$ for $\Sigma_{\mathrm{ACPS}}^{\prime}$ : Given a function $\psi_{i, b}$ and an honestly generated ciphertext $C=(\mathbf{y}, z)$ of $M=b$, it outputs

$$
C^{\prime}:=\left(\mathbf{y}+\left((-1)^{b} p\right) \mathbf{e}_{i}, z\right) \quad \text { for the } i \text {-th unit vector } \mathbf{e}_{i} .
$$

We check that this ciphertext decrypts to $k_{i} \oplus b$ :

$$
\begin{aligned}
& \mathrm{D}_{k}\left(C^{\prime}\right)=\left\lceil\left(\left\langle\mathbf{s} ; \mathbf{y}+\left((-1)^{b} p\right) \mathbf{e}_{i}\right\rangle+z\right) / p\right\rfloor_{p}=\left\lceil\left(\langle\mathbf{s} ; \mathbf{y}\rangle+\left((-1)^{b} p\right) \mathbf{s}_{i}+z\right) / p\right\rfloor_{p} \\
= & \left\lceil\left(\mathbf{s}^{T} \mathbf{A r}+\left((-1)^{b} p\right) \mathbf{s}_{i}+z\right) / p\right\rfloor_{p}=\left\lceil\left(\left((-1)^{b} p\right) \mathbf{s}_{i}-\langle\mathbf{x} ; \mathbf{r}\rangle+p b\right) / p\right\rfloor_{p},=\mathbf{s}_{i} \oplus b .
\end{aligned}
$$

In fact, it is easy to see that ciphertexts $C^{\prime}$ as produced by $\mathcal{F}_{\mathrm{KDM}\left[\Psi^{\prime}\right]}$ are perfectly distributed ciphertexts of $\mathbf{s}_{i} \oplus b$. We get:

Lemma 10. $\mathcal{F}_{\left.\mathrm{KDM}^{\prime} \Psi^{\prime}\right]}$ is a $\mathrm{KDM}\left[\Psi^{\prime}\right]$ oracle in the sense of Theorem 3 .

Lemma 11. The SKE scheme $\Sigma_{\mathrm{ACPS}}^{\prime}$ is IND-CPA secure if the LWE assumption holds for the respective parameters.

A sketch of the proof is contained in the full version of this paper [24].

The Full Scheme $\boldsymbol{\Sigma}_{\text {ACPS }}$. As in the BHHO setting, we can construct the full scheme $\Sigma_{\text {ACPS }}$ with message space $\mathbb{Z}_{p}^{m}$ from $m$ instances of $\Sigma_{\text {ACPS }}^{\prime}$ that use the same public parameters and key in a straightforward manner.

Likewise, by transferring Theorem 9, Theorem 10 and Theorem 11 from $\Sigma_{\text {ACPS }}^{\prime}$ to $\Sigma_{\text {ACPS }}$ and by Theorem 4 , we get

Theorem 12. The $S K E$ scheme $\Sigma_{\mathrm{ACPS}}$ is $\operatorname{RKA-KDM}[\Phi, \Psi]$ secure (for $\Phi$ as defined above in this section and $\Psi$ from the full BHHO scheme) if the LWE assumption holds for the respective parameters.

\subsection{Brakerski-Goldwasser [26]}

In this section we consider the encryption scheme of Brakerski and Goldwasser [26], modified to the symmetric setting. The KDM security of the original (publickey) scheme relies on the hardness of deciding quadratic residuosity in the group $\mathbb{Z}_{N}^{*}$, for Blum integer $N=p \cdot q$. To construct our SKE scheme $\Sigma_{\mathrm{BG}}$ resilient against related key attacks, we additionally have to stipulate that DDH is hard over the subgroup of quadratic residues $\mathrm{QR}_{N}$. We achieve security against the same class of KDM functions as for $\Sigma_{\mathrm{BHHO}}$ from Section 3.1 .

QR Assumption. Let $N$ be a Blum integer of bitlength $\lambda$. With $\mathbb{Z}_{N}^{*}[+1]$ we denote the set of elements in $\mathbb{Z}_{N}^{*}$ with Jacobi symbol +1 and with $\mathrm{QR}_{N}:=$ $\left\{x^{2} \bmod N: x \in \mathbb{Z}_{N}^{*}\right\}$ the set of Quadratic Residues modulo $N$. Then we say that the Quadratic Residuosity (QR) assumption holds in $\mathbb{Z}_{N}^{*}$ if

$$
\left|\operatorname{Pr}\left[\mathcal{A}(N, x)=1: x \leftarrow \mathbb{Z}_{N}^{*}[+1]\right]-\operatorname{Pr}\left[\mathcal{A}(N, x)=1: x \leftarrow \mathrm{QR}_{N}\right]\right|
$$

is negligible for all PPT adversaries $\mathcal{A}$. 
The SKE Scheme $\boldsymbol{\Sigma}_{\mathbf{B G}}^{\prime}$. We define the scheme for messages $M \in\{0,1\}$.

- $\operatorname{Pg}\left(1^{\lambda}\right)$ picks a random Blum integer $N$ of length $\ell(\lambda) 4$ Then samples quadratic residues $g_{1}, \ldots, g_{\lambda} \leftarrow \mathrm{QR}_{N}$ and returns $\pi:=\left(N, g_{1}, \ldots, g_{\lambda}\right)$.

- $\operatorname{Kg}(\pi)$ returns a random bitstring $k \leftarrow\{0,1\}^{\lambda}$.

- $\mathrm{E}_{k}(M)$ picks $r_{1}, \ldots, r_{\lambda} \leftarrow\left[N^{2}\right]$, computes $g_{0}:=\prod_{i \in[\lambda]}\left(g_{i}^{r_{i}}\right)^{-k_{i}}$ and outputs

$$
C:=\left(g_{1}^{r_{1}}, \ldots, g_{\lambda}^{r_{\lambda}},(-1)^{M} \cdot g_{0}\right) \in \mathbb{Z}_{N}^{\lambda+1}
$$

- $\mathrm{D}_{k}(C)$ parses $C$ as $\left(x_{1}, \ldots, x_{\lambda}, y\right)$. Computes $\tilde{M}:=y \cdot \prod_{i \in[\lambda]} x_{i}^{k_{i}}$. Returns 0 if $\tilde{M}=1$, returns 1 if $\tilde{M}=-1$, otherwise returns $\perp$.

The RKA $[\boldsymbol{\Phi}]$ Oracle. The RKA $[\Phi]$ oracle $\mathcal{F}_{\mathrm{RKA}[\Phi]}$ for $\Sigma_{\mathrm{BG}}^{\prime}$ works exactly like the RKA $[\Phi]$ for $\Sigma_{\mathrm{BHHO}}^{\prime}$ from Section 3.1, i.e., $\Phi$ allows for transformations of the secret key under XOR. Analogously to Theorem 5 we have

Lemma 13. $\mathcal{F}_{\mathrm{RKA}[\Phi]}$ is an $\mathrm{RKA}[\Phi]$ oracle for $\Sigma_{\mathrm{BG}}^{\prime}$ in the sense of Theorem 2.

The $\operatorname{KDM}\left[\Psi^{\prime}\right]$ Oracle. Analogously to $\Sigma_{\mathrm{BHHO}}^{\prime}$ we define

$$
\Psi^{\prime}:=\left\{\psi_{i, b}:\{0,1\}^{\lambda} \rightarrow\{0,1\}, k \mapsto k_{i} \oplus b: i \in[\lambda], b \in\{0,1\}\right\}
$$

Given a function $\psi_{i, b}$ and a ciphertext $C=\left(x_{1}, \ldots, x_{\lambda}, y\right)$, the $\mathrm{KDM}\left[\Psi^{\prime}\right]$ oracle $\mathcal{F}_{\mathrm{KDM}\left[\Psi^{\prime}\right]}$ for $\Sigma_{\mathrm{BG}}^{\prime}$ simply returns

$$
C^{\prime}:=\left(x_{1}^{\prime}, \ldots, x_{\lambda}^{\prime}, y^{\prime}\right):=\left(x_{1}, \ldots, x_{i-1},(-1) \cdot x_{i}, x_{i+1}, \ldots, x_{\lambda}, y\right)
$$

We check that this decrypts to $k_{i} \oplus b$ if $\mathcal{F}_{\mathrm{KDM}\left[\Psi^{\prime}\right]}$ is given an honestly generated ciphertext of $b$ (the constant part of $\psi_{i, b}$ ), i.e., $y=(-1)^{b} \cdot \prod_{j \in[\lambda]} x_{j}{ }^{-k_{j}}$ :

$\mathrm{D}_{k}\left(C^{\prime}\right)=y^{\prime} \cdot \prod_{j \in[\lambda]} x_{j}^{\prime k_{j}} \stackrel{(*)}{=} y \cdot(-1)^{k_{i}} \cdot \prod_{j \in[\lambda]} x_{j}^{k_{j}}=(-1)^{b+k_{i}} \cdot \prod_{j \in[\lambda]} x_{j}{ }^{-k_{j}} \cdot x_{j}{ }^{k_{j}}=(-1)^{k_{i} \oplus b}$ $(*)$ since $x_{i}^{\prime}=(-1) \cdot x_{i}$ and $x_{j}^{\prime}=x_{j}$ for $j \in[\lambda] \backslash\{i\}$.

Lemma 14. $\mathcal{F}_{\mathrm{KDM}\left[\Psi^{\prime}\right]}$ is a $\mathrm{KDM}\left[\Psi^{\prime}\right]$ oracle for $\Sigma_{\mathrm{BG}}^{\prime}$ in the sense of Theorem 3 if $Q R$ is hard in the underlying group $\mathbb{Z}_{N}^{*}$.

Proof. To show the indistinguishability of $\mathcal{F}_{\mathrm{KDM}\left[\Psi^{\prime}\right]}$ 's output we use the interactive vector game (IV) from [26], Section 5. In the interactive $\lambda$-vector game the experiment picks a Blum integer $N$, a quadratic residues $g_{1}, \ldots, g_{\lambda} \leftarrow \mathrm{QR}_{N}$ and a bit $b \leftarrow\{0,1\}$ and sends $N, g_{1}, \ldots, g_{\lambda}$ to a PPT adversary $\mathcal{A}$ that has to guess $b$. It then provides $\mathcal{A}$ with an oracle that, given a query $\mathbf{a} \in\{0,1\}^{\lambda}$, returns

${ }^{4}$ We use $\ell(\lambda)$ here since the IND-CPA security of Brakerski and Goldwasser's original scheme requires that $N$ is substantially shorter than the number of components/key length $\lambda$, e.g., $\ell(\lambda)=\lambda / 2$. We refer to [26], Theorem 6.1 for details. 
$\left((-1)^{\mathbf{a}_{1}} g_{1}^{r}, \ldots,(-1)^{\mathbf{a}_{\lambda}} g_{\lambda}^{r}\right)$ if $b=0$ and $\left(g_{1}^{r}, \ldots, g_{\lambda}^{r}\right)$ if $b=1$ for fresh randomness $r$. [26] show that $\mathcal{A}$ 's advantage is negligible if the QR assumption holds in $\mathbb{Z}_{N}^{*}$. Let $\mathcal{D}$ be a PPT algorithm to distinguish $\mathcal{F}_{\mathrm{KDM}\left[\Psi^{\prime}\right]}\left(\psi, \mathrm{E}_{k}(M)\right)$ from $\mathrm{E}_{k}(\psi(k))$ in the sense of Theorem 3. We construct an adversary $\mathcal{S}$ on the interactive 1vector game that utilizes $\mathcal{D}$ : First, $\mathcal{S}$ sets $\pi$ to the parameters $\left(N, g_{1}, \ldots, g_{\lambda}\right)$ received from the interactive $\lambda$-vector game, samples a key $k \leftarrow\{0,1\}^{\lambda}$ and then sends $\pi$ and $k$ to $\mathcal{D}$. For each query $\psi_{i, b}$ received from $\mathcal{D}, \mathcal{S}$ picks randomness $r_{1}, \ldots, r_{i-1}, r_{i+1}, \ldots, r_{\lambda} \leftarrow\left[N^{2}\right]$ and queries the interactive $\lambda$-vector game with vector $\mathbf{a} \in\{0,1\}^{\lambda}$ where $\mathbf{a}_{i}:=1$ and $\mathbf{a}_{j}:=0$ for $j \neq i$. $\mathcal{S}$ gets a response $\left(x_{1}, \ldots, x_{\lambda}\right)$ and sets $x_{i}^{\prime}:=x_{i}$ and $x_{j}^{\prime}:=x_{j}^{r_{j}}$ for $j \neq r$. It then sends $\left(x_{1}^{\prime}, \ldots, x_{\lambda}^{\prime},(-1)^{b} \cdot \prod_{j \in[\lambda]} x_{j}^{\prime-k_{j}}\right)$ to $\mathcal{D}$. It is easy to check that this equals $\mathcal{F}_{\left.\mathrm{KDM}^{\prime} \Psi^{\prime}\right]}\left(\psi_{i, b}, \mathrm{E}_{k}(b ; \hat{r})\right)$ if the bit picked by the $\lambda$-vector game is 0 , or $\mathrm{E}_{k}(\psi(k) ; \hat{r})$ otherwise (where randomness $\left.\hat{r}:=\left(r r_{1}, \ldots, r_{i-1}, r, r_{i+1}, \ldots, r r_{\lambda}\right)\right)$.

The advantage of $\mathcal{S}$ is the advantage of $\mathcal{D}$ at the same asymptotic time complexity. Thus, if $\mathrm{QR}$ holds in $\mathbb{Z}_{N}^{*}$, no such adversary $\mathcal{D}$ with non-negligible advantage can exist.

Lemma 15. The $S K E$ scheme $\Sigma_{\mathrm{BG}}^{\prime}$ is IND-CPA secure if $Q R$ is hard over thegroup $\mathbb{Z}_{N}^{*}$ and $D D H$ is hard over the subgroup of quadratic residues $\mathrm{QR}_{N}$.

Proof. This proof is completely analogous to the IND-CPA proof for $\Sigma_{\text {BHHO }}^{\prime}$ (see Theorem 7). We first collapse the randomness to one random exponent per ciphertext. For this we rely on the hardness of $\mathrm{DDH}$ over $\mathrm{QR}_{N}$. Subsequently we utilize the IND-CPA security of Brakerski and Goldwasser's original scheme to conclude the proof.

The Full Scheme $\boldsymbol{\Sigma}_{\mathrm{BG}}$. Analogously to the setting for BHHO (Section 3.1), we can canonically construct the full scheme $\Sigma_{\mathrm{BG}}$ for message space $\{0,1\}^{\lambda}$ from $\lambda$ instances of $\Sigma_{\mathrm{BG}}^{\prime}$ using the same public parameters and the same key. The class of RKA functions remains the same, while the class of KDM functions automatically extends from $\Psi^{\prime}$ to

$$
\Psi:=\left\{\psi_{\mathbf{i}, M}:\{0,1\}^{\lambda} \rightarrow\{0,1\}^{\lambda}, k \mapsto\left(k_{\mathbf{i}_{1}} \oplus \Delta_{1}, \ldots, k_{\mathbf{i}_{\lambda}} \oplus \Delta_{\lambda}\right): \mathbf{i} \in[\lambda]^{\lambda}, \Delta \in\{0,1\}^{\lambda}\right\}
$$

Since we can canonically transfer Theorem 13, Theorem 14 and Theorem 15 from $\Sigma_{\mathrm{BG}}^{\prime}$ to $\Sigma_{\mathrm{BG}}$ we get the final result of this section by Theorem 4

Theorem 16. The SKE scheme $\Sigma_{\mathrm{BG}}$ is $\operatorname{RKA-KDM}[\Phi, \Psi]$ secure (for $\Phi$ and $\Psi$ as defined above in this section) if $Q R$ is hard in the underlying group $\mathbb{Z}_{N}^{*}$ and $D D H$ is hard over the subgroup of quadratic residues $\mathrm{QR}_{N}$.

\subsection{Bellare et al. [14}

Since Applebaum's work on KDM amplification [4], it is known that projectionKDM security implies bounded-KDM security. Projection-KDM security allows for KDM functions where each output bit depends only on one input bit (key 
bit). Bounded-KDM security means that the class of KDM functions is the set of all functions that can be represented by a circuit of bounded size $L$. We refer to this function class as $\Psi_{\text {bnd }(L)}$ from now on. To our knowledge, currently the most efficient way to construct a bounded-KDM secure scheme from a projection-KDM secure one is the approach of Bellare, Hoang, and Rogaway [14] (henceforth BHR). In this section we observe that their construction also maintains RKA security in our sense. Thus, we can plug all of our projectionKDM secure schemes (i.e., $\Sigma_{\mathrm{BG}}, \Sigma_{\mathrm{ACPS}}$ and $\Sigma_{\mathrm{BHHO}}$ ) into their framework to get RKA-bounded-KDM secure schemes. Obviously, this result holds for any projection-KDM secure scheme that is RKA secure (with a suitable oracle in our sense).

(Projective) Garbling Schemes. What follows is a quick introduction to garbling schemes established by [14]. A garbling scheme is a tuple of algorithms ( $\left.\mathrm{GC}_{\text {garble }}, \mathrm{GC}_{\text {encode }}, \mathrm{GC}_{\text {decode }}, \mathrm{GC}_{\text {eval }}\right) 5$ The algorithm $\mathrm{GC}_{\text {garble }}$ is probabilistic while the remaining algorithms are deterministic. Given an encoding of the security parameter and a function $f, \mathrm{GC}_{\text {garble }}\left(1^{\lambda}, f\right)$ outputs the description of a garbled circuit $(F, e, d)$. Here, $F$ is a function mapping garbled inputs to garbled outputs. E.g., $F$ could be a circuit in terms of gates and wires together with a garbled table for each gate. The outputs $e$ and $d$ contain information to encode and decode the input and output of $F$ respectively. We say that a garbling scheme is correct if $\mathrm{GC}_{\text {decode }}\left(d, \mathrm{GC}_{\text {eval }}\left(F, \mathrm{GC}_{\text {encode }}(M, e)\right)\right)=f(M)$ for all functions $f$ (from a certain class), inputs $M \in\{0,1\}^{\lambda}$ and descriptions $(F, e, d) \leftarrow \mathrm{GC}_{\text {garble }}\left(1^{\lambda}, f\right)$ of garbled circuits for $f$.

For our application we need so-called projective garbling schemes. Basically, a garbling scheme is projective if for all $\mathbf{x}:=\mathrm{GC}_{\text {encode }}(e, M)$ and $\mathbf{x}^{\prime}:=$ $\mathrm{GC}_{\text {encode }}\left(e, M^{\prime}\right)$, we have $\left|\mathbf{x}_{i}\right|=\left|\mathbf{x}_{i}^{\prime}\right|$ for $i \in[\lambda]$ and $\mathbf{x}_{i}=\mathbf{x}_{i}^{\prime}$ for $i \in[\lambda]$ with $M_{i}=M_{i}^{\prime}$ (see [15] for a rigorous definition). One well-known way to construct a projective garbling scheme is to assign a pair of keys to each wire corresponding to low and high voltage $(0 / 1)$ respectively. Then $e$ is a tuple of pairs of keys and $\mathrm{GC}_{\text {encode }}(M, e)$ picks the keys from $e$ corresponding to the bits of $M$.

Furthermore, we say that a garbling scheme is privacy preserving if for any two (adversarially chosen) functions $f_{0}, f_{1}$ with the same circuit size and inputs $x_{0}, x_{1}$ of same length with $f_{0}\left(x_{0}\right)=f_{1}\left(x_{1}\right)$, no adversary can distinguish $\left(F_{0}, \mathrm{GC}_{\text {encode }}\left(e_{0}, x_{0}\right), d_{0}\right)$ from $\left(F_{1}, \mathrm{GC}_{\text {encode }}\left(e_{1}, x_{1}\right), d_{1}\right)$ (where $\left(F_{b}, e_{b}, d_{b}\right)$ $\left.\leftarrow \mathrm{GC}_{\text {garble }}\left(1^{\lambda}, f_{b}\right), b \in\{0,1\}\right)$. We refer to [15] for a more detailed definition.

The Construction of BHR. The construction creates a symmetric $\operatorname{KDM}\left[\Psi_{\text {bnd }(L)}\right]$-secure encryption scheme $\Sigma_{\mathrm{BHR}}=(\mathrm{Pg}, \mathrm{Kg}, \mathrm{E}, \mathrm{D})$ from any projection-KDM-secure encryption scheme $\Sigma^{\prime}=\left(\mathrm{Pg}^{\prime}, \mathrm{Kg}^{\prime}, \mathrm{E}^{\prime}, \mathrm{D}^{\prime}\right)$ and any privacy preserving projective garbling scheme $\left(\mathrm{GC}_{\text {garble }}, \mathrm{GC}_{\text {encode }}, \mathrm{GC}_{\text {decode }}, \mathrm{GC}_{\text {eval }}\right)$ as follows.

\footnotetext{
${ }^{5}$ For simplicity we omit the additional evaluation function from [14] and restrict to inputs of length $\lambda$ here.
} 
$-\operatorname{Pg}\left(1^{\lambda}\right)$ returns $\operatorname{Pg}^{\prime}\left(1^{\lambda}\right)$.

- $\mathrm{Kg}(\pi)$ returns $\mathrm{Kg}^{\prime}(\pi)$.

- $\mathrm{E}_{k}(M)$ first generates a garbled circuit for the identity function $\operatorname{ID}_{\lambda}$ on bitstrings of length $\lambda:(F, e, d) \leftarrow \mathrm{GC}_{\text {garble }}\left(1^{\lambda}, \mathrm{ID}_{\lambda}\right)$. It then encodes the message $\mathbf{x}:=\mathrm{GC}_{\text {encode }}(e, M)$ (w.l.o.g. $\mathbf{x} \in\{0,1\}^{\lambda \times \lambda}$ ). Finally, it outputs the ciphertext $C:=\left(F, d, \mathrm{E}_{k}^{\prime}\left(\mathbf{x}_{i}\right)\right)$.

- $\mathrm{D}_{k}\left(\left(F, d,\left(\mathbf{c}_{i}\right)_{i \in[\lambda]}\right)\right)$ first decrypts the keys for the input wires $\mathbf{x}_{i}:=\mathrm{D}_{k}^{\prime}\left(\mathbf{c}_{i}\right)$ and then evaluates the circuit to compute and output the message $M:=$ $\mathrm{GC}_{\text {decode }}\left(d, \mathrm{GC}_{\text {eval }}(F, \mathbf{x})\right)$.

An RKA $[\boldsymbol{\Phi}]$ oracle for $\boldsymbol{\Sigma}_{\mathrm{BHR}}$. Given an RKA $[\Phi]$ oracle $\mathcal{F}_{\mathrm{RKA}[\Phi]}^{\prime}$ for $\Sigma^{\prime}$, we can construct an RKA $[\Phi]$ oracle $\mathcal{F}_{\mathrm{RKA}[\Phi]}$ for $\Sigma_{\mathrm{BHR}}$ (note that we maintain the class of RKA functions). Let $C=\left(F, d,\left(\mathbf{c}_{i}\right)_{i \in[\lambda]}\right)$ be an honestly generated ciphertext and $\varphi \in \Phi$ be an RKA function. We define $\mathcal{F}_{\mathrm{RKA}[\Phi]}(C):=\left(F, d,\left(\mathcal{F}_{\mathrm{RKA}[\Phi]}^{\prime}\left(\mathbf{c}_{i}\right)\right)_{i \in[\lambda]}\right)$. A straightforward hybrid argument over the $\mathbf{c}_{i}$, based on the indistinguishability of $\mathcal{F}_{\mathrm{RKA}[\Phi]}^{\prime}$, shows the indistinguishability of $\mathcal{F}_{\mathrm{RKA}[\Phi]}(C)$.

Theorem 17. Let $\Sigma^{\prime}$ be a RKA-KDM $[\Phi, \Psi]$-secure SKE scheme with an indistinguishable $\mathrm{RKA}[\Phi]$ oracle $\mathcal{F}_{\mathrm{RKA}[\Phi]}$. If $\Psi$ covers projections, then $\Sigma_{\mathrm{BHR}}$ is an $\operatorname{RKA}-\operatorname{KDM}\left[\Phi, \Psi_{\mathrm{bnd}(L)}\right]$-secure $S K E$ for any arbitrary but fixed bound $L$.

Proof. We only sketch the proof here, which is straightforward and based on a short sequence of games. Our first game is the original RKA-KDM $[\Phi, \Psi]$ experiment (see Theorem 1). In the next game, we no longer use the secret key itself to answer the RKA part of queries. More concretely, for a given RKA-KDM query $(\varphi, \psi)$, we compute $C \leftarrow \mathrm{E}_{k}(\psi(k))$ and output $\mathcal{F}_{\mathrm{RKA}[\Phi]}(\varphi, C)$ instead of directly returning $\mathrm{E}_{\varphi(k)}(\psi(k))$. The indistinguishability of this game hop follows directly from the indistinguishability of RKA $[\Phi]$. Finally, we can simply follow the strategy from [15], Theorem 15, to compute $C$. This strategy requires that the garbling scheme used to construct $\Sigma_{\mathrm{BHR}}$ is privacy preserving and projective.

Acknowledgements. The authors would like to thank Martijn Stam for useful discussions and Rafael Dowsley for kindling our interest in the topic. Furthermore, we would like to thank Viet Tung Hoang for pointing out a more efficient and less complicated way to achieve bounded-KDM security (based on [14]) than the one we first decided on (based on [6] ).

\section{References}

[1] Adão, P., Bana, G., Herzog, J.C., Scedrov, A.: Soundness of Formal Encryption in the Presence of Key-Cycles. In: de Capitani di Vimercati, S., Syverson, P.F., Gollmann, D. (eds.) ESORICS 2005. LNCS, vol. 3679, pp. 374-396. Springer, Heidelberg (2005)

[2] Akavia, A., Goldwasser, S., Vaikuntanathan, V.: Simultaneous Hardcore Bits and Cryptography against Memory Attacks. In: Reingold, O. (ed.) TCC 2009. LNCS, vol. 5444, pp. 474-495. Springer, Heidelberg (2009) 
[3] Applebaum, B.: Garbling XOR gates "For free" in the standard model. In: Sahai, A. (ed.) TCC 2013. LNCS, vol. 7785, pp. 162-181. Springer, Heidelberg (2013)

[4] Applebaum, B.: Key-Dependent Message Security: Generic Amplification and Completeness. In: Paterson, K.G. (ed.) EUROCRYPT 2011. LNCS, vol. 6632, pp. 527-546. Springer, Heidelberg (2011)

[5] Applebaum, B., Cash, D., Peikert, C., Sahai, A.: Fast Cryptographic Primitives and Circular-Secure Encryption based on Hard Learning Problems. In: Halevi, S. (ed.) CRYPTO 2009. LNCS, vol. 5677, pp. 595-618. Springer, Heidelberg (2009)

[6] Barak, B., Haitner, I., Hofheinz, D., Ishai, Y.: Bounded Key-Dependent Message Security. In: Gilbert, H. (ed.) EUROCRYPT 2010. LNCS, vol. 6110, pp. 423-444. Springer, Heidelberg (2010)

[7] Bellare, M., Cash, D.: Pseudorandom Functions and Permutations Provably Secure against Related-Key Attacks. In: Rabin, T. (ed.) CRYPTO 2010. LNCS, vol. 6223, pp. 666-684. Springer, Heidelberg (2010)

[8] Bellare, M., Keelveedhi, S.: Authenticated and Misuse-Resistant Encryption of Key-Dependent Data. In: Rogaway, P. (ed.) CRYPTO 2011. LNCS, vol. 6841, pp. 610-629. Springer, Heidelberg (2011)

[9] Bellare, M., Kohno, T.: A Theoretical Treatment of Related-Key Attacks: RKAPRPs, RKA-PRFs, and Applications. In: Biham, E. (ed.) EUROCRYPT 2003. LNCS, vol. 2656, pp. 491-506. Springer, Heidelberg (2003)

[10] Bellare, M., Brakerski, Z., Naor, M., Ristenpart, T., Segev, G., Shacham, H., Yilek, S.: Hedged Public-Key Encryption: How to Protect against Bad Randomness. In: Matsui, M. (ed.) ASIACRYPT 2009. LNCS, vol. 5912, pp. 232-249. Springer, Heidelberg (2009)

[11] Bellare, M., Hofheinz, D., Yilek, S.: Possibility and Impossibility Results for Encryption and Commitment Secure under Selective Opening. In: Joux, A. (ed.) EUROCRYPT 2009. LNCS, vol. 5479, pp. 1-35. Springer, Heidelberg (2009)

[12] Bellare, M., Cash, D., Keelveedhi, S.: Ciphers that Securely Encipher their own Keys. In: ACM Conference on Computer and Communications Security, pp. 423432 (2011)

[13] Bellare, M., Cash, D., Miller, R.: Cryptography Secure against Related-Key Attacks and Tampering. In: Lee, D.H., Wang, X. (eds.) ASIACRYPT 2011. LNCS, vol. 7073, pp. 486-503. Springer, Heidelberg (2011)

[14] Bellare, M., Hoang, V.T., Rogaway, P.: Foundations of garbled circuits. In: Proceedings of the 2012 ACM Conference on Computer and Communications Security, pp. 784-796. ACM (2012)

[15] Bellare, M., Hoang, V.T., Rogaway, P.: Foundations of garbled circuits. Cryptology ePrint Archive, Report 2012/265 (2012), http://eprint.iacr.org/

[16] Biham, E.: New types of Cryptoanalytic Attacks using Related Keys. In: Helleseth, T. (ed.) EUROCRYPT 1993. LNCS, vol. 765, pp. 398-409. Springer, Heidelberg (1994)

[17] Biham, E., Dunkelman, O., Keller, N.: A Related-Key Rectangle Attack on the Full KASUMI. In: Roy, B. (ed.) ASIACRYPT 2005. LNCS, vol. 3788, pp. 443-461. Springer, Heidelberg (2005)

[18] Biham, E., Dunkelman, O., Keller, N.: Related-Key Impossible Differential Attacks on 8-Round AES-192. In: Pointcheval, D. (ed.) CT-RSA 2006. LNCS, vol. 3860, pp. 21-33. Springer, Heidelberg (2006)

[19] Biham, E., Dunkelman, O., Keller, N.: A Simple Related-Key Attack on the Full SHACAL-1. In: Abe, M. (ed.) CT-RSA 2007. LNCS, vol. 4377, pp. 20-30. Springer, Heidelberg (2006) 
[20] Biryukov, A., Khovratovich, D.: Related-Key Cryptanalysis of the Full AES-192 and AES-256. In: Matsui, M. (ed.) ASIACRYPT 2009. LNCS, vol. 5912, pp. 1-18. Springer, Heidelberg (2009)

[21] Biryukov, A., Khovratovich, D., Nikolić, I.: Distinguisher and Related-Key Attack on the Full AES-256. In: Halevi, S. (ed.) CRYPTO 2009. LNCS, vol. 5677, pp. 231-249. Springer, Heidelberg (2009)

[22] Biryukov, A., Dunkelman, O., Keller, N., Khovratovich, D., Shamir, A.: Key Recovery Attacks of Practical Complexity on AES-256 Variants with up to 10 Rounds. In: Gilbert, H. (ed.) EUROCRYPT 2010. LNCS, vol. 6110, pp. 299-319. Springer, Heidelberg (2010)

[23] Black, J., Rogaway, P., Shrimpton, T.: Encryption-Scheme Security in the Presence of Key-Dependent Messages. In: Nyberg, K., Heys, H.M. (eds.) SAC 2002. LNCS, vol. 2595, pp. 62-75. Springer, Heidelberg (2003)

[24] Böhl, F., Davies, G.T., Hofheinz, D.: Encryption schemes secure under related-key and key-dependent message attacks. IACR Cryptology ePrint Archive 653 (2013)

[25] Boneh, D., Halevi, S., Hamburg, M., Ostrovsky, R.: Circular-secure encryption from Decision Diffie-Hellman. In: Wagner, D. (ed.) CRYPTO 2008. LNCS, vol. 5157, pp. 108-125. Springer, Heidelberg (2008)

[26] Brakerski, Z., Goldwasser, S.: Circular and Leakage Resilient Public-Key Encryption under Subgroup Indistinguishability - (or: Quadratic Residuosity strikes back). In: Rabin, T. (ed.) CRYPTO 2010. LNCS, vol. 6223, pp. 1-20. Springer, Heidelberg (2010)

[27] Brakerski, Z., Goldwasser, S., Kalai, Y.T.: Black-Box Circular-Secure Encryption beyond Affine Functions. In: Ishai, Y. (ed.) TCC 2011. LNCS, vol. 6597, pp. 201218. Springer, Heidelberg (2011)

[28] Camenisch, J., Chandran, N., Shoup, V.: A Public Key Encryption Scheme Secure against Key Dependent Chosen Plaintext and Adaptive Chosen ciphertext Attacks. In: Joux, A. (ed.) EUROCRYPT 2009. LNCS, vol. 5479, pp. 351-368. Springer, Heidelberg (2009)

[29] Dziembowski, S., Pietrzak, K.: Leakage-Resilient Cryptography. In: FOCS, pp. 293-302 (2008)

[30] Goldwasser, S., Micali, S.: Probabilistic Encryption. J. Comput. Syst. Sci. 28(2), 270-299 (1984)

[31] Halevi, S., Krawczyk, H.: Security under Key-Dependent Inputs. In: ACM Conference on Computer and Communications Security, pp. 466-475 (2007)

[32] Hofheinz, D.: Circular Chosen-Ciphertext Security with Compact Ciphertexts. In: Johansson, T., Nguyen, P.Q. (eds.) EUROCRYPT 2013. LNCS, vol. 7881, pp. 520-536. Springer, Heidelberg (2013)

[33] Hofheinz, D., Unruh, D.: Towards Key-Dependent Message Security in the Standard Model. In: Smart, N.P. (ed.) EUROCRYPT 2008. LNCS, vol. 4965, pp. 108-126. Springer, Heidelberg (2008)

[34] Malkin, T., Teranishi, I., Yung, M.: Efficient Circuit-Size Independent Public Key Encryption with KDM Security. In: Paterson, K.G. (ed.) EUROCRYPT 2011. LNCS, vol. 6632, pp. 507-526. Springer, Heidelberg (2011)

[35] Micali, S., Reyzin, L.: Physically Observable Cryptography. In: Naor, M. (ed.) TCC 2004. LNCS, vol. 2951, pp. 278-296. Springer, Heidelberg (2004)

[36] Wee, H.: Public Key Encryption against Related Key Attacks. In: Fischlin, M., Buchmann, J., Manulis, M. (eds.) PKC 2012. LNCS, vol. 7293, pp. 262-279. Springer, Heidelberg (2012) 